\title{
Influence of the finite ionospheric conductivity on dispersive, nonradiative field line resonances
}

\author{
A. Streltsov, W. Lotko \\ Received: 23 February 1996 / Revised: 28 October 1996 / Accepted: 13 January 1997
}

\begin{abstract}
The influence of the finite ionospheric conductivity on the structure of dispersive, nonradiative field line resonances (FLRs) is investigated for the first four odd harmonics. The results are based on a linear, magnetically incompressible, reduced, two-fluid MHD model. The model includes effects of finite electron inertia (at low altitude) and finite electron pressure (at high altitude). The ionosphere is treated as a highintegrated conducting substrate. The results show that even very low ionospheric conductivity $\left(\Sigma_{P}=2 \mathrm{mho}\right)$ is not sufficient to prevent the formation of a largeamplitude, small-scale, nonradiative FLR for the third and higher harmonics when the background transverse plasma inhomogeneity is strong enough. At the same time, the fundamental FLR is strongly affected by a state of low conductivity, and when $\Sigma_{P}=2 \mathrm{mho}$, this resonance forms only small-amplitude, relatively broad electromagnetic disturbance. The difference in conductivities of northern and southern ionospheres does not produce significant asymmetry in the distribution of electric and magnetic fields along the resonant field line. The transverse gradient of the background Alfvén speed plays an important role in structure of the FLR when the ionospheric conductivity is finite. In cases where the transverse inhomogeneity of the plasma is not strong enough, the low ionospheric conductivity can prevent even higher-harmonic FLRs from contracting to small scales where dispersive effects are important. The application of these results to the formation and temporal evolution of small-scale, active auroral arc forms is discussed.
\end{abstract}

\section{Introduction}

Field line resonance (FLR) is possible at any location in the Earth's magnetosphere where the ambient magnetic

Correspondence to: A. Streltsov field lines are closed and a transverse gradient in the background Alfvén speed $v_{A}$ exists. (Southwood, 1974; Chen and Hasegawa, 1974). The resonance occurs on magnetic field lines where the frequency of the driven oscillation matches the eigenfrequency of a shear Alfvén wave standing along a magnetic shell between two conducting ionospheres. In one-fluid MHD, the northsouth electric and east-west magnetic fields have a singularity at the resonant surface. This singularity is resolved when two-fluid dispersive effects connected with the finite ion Larmor radius, finite electron pressure ("kinetic" dispersion), or finite electron inertia ("inertial" dispersion), alone or in combination, are included (Hasegawa, 1976; Goertz and Boswell, 1979). Actually, both types of dispersion are relevant for Alfvén waves propagating or standing between the two auroral ionospheres. In the hot plasma of the equatorial magnetosphere, kinetic effects are dominant, whereas at low altitude, near the ionosphere, inertial dispersion dominates. The transition from inertial to kinetic regimes occurs approximately midway along a field line between the ionosphere and equatorial plane where $v_{A}=v_{T e}$, and $v_{T e}$ is the electron thermal speed (Lysak and Carlson, 1981).

The presence of dispersion may lead to saturation of the amplitude and limitation in the contraction of the transverse scale-size of the resonant solution when the dispersion promotes radiation of dispersive Alfvén waves. According to their dispersive properties these waves propagate energy perpendicular to the ambient magnetic field in the direction of the transverse gradient in the background Alfvén speed when the inertial dispersion dominates, and in the opposite direction, when kinetic effects are more important (Inhester, 1987; Wei et al., 1994). As discussed by Streltsov and Lotko (1995) (referred to as SL1), the tendency of the "radiated" dispersive Alfvén wave to propagate energy away from the resonance is thwarted when,on its transit between ionospheres, the perpendicular group propagation averages to zero. Counteracting effects of the two different types of dispersion at different altitudes along the 
resonant field line can therefore cause the wave energy to become trapped inside the resonance layer. The fine structure of such nonradiative, dispersive FLR layers, along the resonant field line as well as perpendicular to it, was investigated in detail by Streltsov and Lotko (1996) (referred to as SL2) for the first four odd harmonics.

In this and in our previous papers the balance between counteraction of kinetic and inertial dispersions during the wave transit between ionospheric boundaries, which provides nonradiative condition, is attained for each harmonic and for some particular values of $B_{0}$ and $T_{e 0}$ (subscript 0 indicates value at the equator), by adjustment of the background Alfvén speed along the resonant field line. But we wish to emphasize that the seemingly contrived choice of background parameters yielding a nonradiative condition reflects the fact that we are modeling only a limited range of magnetic shells and a monochromatic generator. In general, dispersive, nonradiative FLRs are an unavoidable consequence of magnetospheric inhomogeneity. On relatively low magnetic shells, where the Alfvén speed is relatively large because of the intense dipole magnetic field, the net Alfvén wave dispersion along field lines tends to be dominated by the electron inertia. On relatively high $\mathrm{L}$ shells, in particular, those for which a substantial portion of the flux tube threads the hot plasma sheet, the Alfvén wave dispersion, averaged along the field line, is dominantly kinetic. Because the magnetosphere is a continuous but spatially varying medium, conditions for nonradiative and weakly radiative resonances will be met over some range of intermediate $\mathrm{L}$ shells between the two extremes. The most intense dispersive Alfvén waves are expected on these L shells. For the scope of this study we do not attach much significance to the exact location of the nonradiative resonance shell; to determine the precise location, for given magnetospheric conditions, more realistic models for the ambient magnetic field, background plasma density and temperature must be used. It is reasonable to assume, however, that the likely location lies within the auroral zone, where the most intense, low altitude electric fields, which we associate with dispersive Alfvén waves, are usually found.

The main conclusions derived from our previous studies (SL1, 2) are: (1) large-amplitude, narrow resonances are likely to be found at steep transverse gradients in the background Alfvén speed; (2) the coupling of energy from external sources to the resonance magnetic shell via surface waves is more effective when the azimuthal wave number is small; (3) in nonradiative FLRs wave energy is focused at low altitudes where the background Alfven speed is the largest; (4) the characteristic transverse size of the resonance tends to be smaller for higher harmonics; and (5) the field-aligned potential drop in nonradiative dispersive FLRs may be as large as several kilovolts and is likely to produce accelerated electrons leading to kilometer scale and smaller, discrete auroral arcs. All of these results were obtained for the approximations in which FLRs occur on the magnetic field lines bounded by two perfectly conducting ionospheres. Results from the previous studies, as well as the one reported here, we also obtained by choosing ambient plasma and magnetic field parameters representative of the inner edge of the nightside plasma sheet. This choice of parameters was made for three reasons: (1) steep transverse gradients in the background Alfvén speed are often observed here (Hughes and Grard, 1984; Persoon et al., 1988; SL1); (2) satellite measurements show that localized, largeamplitude, small-scale transverse electric fields, resembling those of the nonradiative, dispersive Alfvén waves, occur predominantly on the same nightside, auroral magnetic field lines (Bennett et al., 1983). (3) this region also maps along magnetic field lines to that part of the nightside auroral oval where the most intense, bright and narrow auroral arcs are typically observed (Akasofu, 1994). Therefore, this region is of particular interest for understanding processes of ionospheremagnetosphere coupling.

The approximation of almost perfectly conducting ionospheres in our previous studies was made to isolate amplitude limiting effects attributed to dispersion. In this study, we now consider the combined influence of dispersion and finite ionospheric conductivity on the formation and temporal evolution of FLR layers. At least two questions arise in this situation. (1) Can the finite ionospheric conductivity prevent resonances from contracting to small scales where dispersive effects become important? (2) What are the quantitative effects of finite conductivity on dispersive FLRs (amplitude variation, scale size, resonance location, etc.)?

In this work, variations in the finite ionospheric conductivity and in the steepness of the transverse plasma inhomogeneity are considered, and their influence on the fine structure and amplitude of dispersive, nonradiative FLRs is investigated for the first four odd harmonics. The results are obtained from computations based on a linear, magnetically incompressible, twofluid MHD model. The model is described in detail in SL1 and SL2; and a brief summary of it is given in the next section. Section 2 also describes the model of the finite ionospheric conductivity used in the computations. The numerical method, parameters of the background plasma inhomogeneity and boundary generator are briefly described in Sec. 3. Section 4 contains the discussion of numerical results and their possible connection with observed auroral phenomena.

\section{Theory}

\subsection{Reduced two-fluid model}

In this study the inner edge of the nightside plasmasheet will be modeled as an inhomogeneous, magnetized, collisionless, low- $\beta$ plasma $\left(\beta=8 \pi P_{0} / B_{0}^{2}\right.$ and $P_{0}$ is the plasma gas pressure), initially free of bulk flows and electrical currents. The following hierarchy of small parameters $(\epsilon \ll 1)$ is used in the derivation of the reduced two-fluid MHD model for ultra-low-frequency oscillations given later (Chmyrev et al., 1988; SL2):

$\epsilon^{2} \sim \omega / \omega_{c i} \sim\left(\rho_{i} / l_{\perp}\right)^{2} \sim\left(v_{E} / v_{T i}\right)^{2} \sim\left(l_{\perp} / l_{\|}\right)^{2}$. 
Here $\omega_{c i}$ is the ion gyrofrequency, $v_{T i}$ is the ion thermal speed, $v_{E}$ is the electric drift speed, $\rho_{i}$ is the ion Larmour radius, and $l_{\perp}$ and $l_{\|}$are the characteristic scale sizes of the solution in the directions locally perpendicular and parallel to the ambient magnetic field.

For simplicity, the background magnetic field $\mathbf{B}_{0}$ is taken to be uniform throughout the region of interest, and the simulation domain can be considered as a straightened out auroral flux tube with finite conducting ionospheres forming the top and bottom boundaries. In this geometry the coordinate system is as follows: the $z$ axis is along the ambient magnetic field $\left(\mathbf{B}_{0}=\hat{\mathbf{z}} B_{0}\right)$; the $x$ axis points opposite to the perpendicular gradient in the background Alfvén speed; and the $y$ axis, points in the west-east (along local time) direction, completing the right-hand system.

The reduced, two-fluid MHD model, on which the study will be based, includes equations describing the electron parallel momentum, electron mass continuity, and current continuity combined with ion momentum. The low $\beta$ of the plasma, together with this ordering of small parameters, allows a characterization of the magnetically incompressible perturbations described by the model in terms of three scalar functions: an electric potential $\phi$, the quasi-neutral density perturbation $n$, and a magnetic flux function $A$, corresponding to the parallel component of the perturbed vector potential (Kadomtsev, 1965).

Because FLRs and associated auroral arcs are very narrow in latitude and practically homogeneous in local time (Greenwald and Walker, 1980; Samson et al., 1992), the azimuthal variation of the solutions can be ignored $\left(\partial_{y}=0\right)$ in the lowest approximation. The twodimensionality of the problem then eliminates the strong vector $(\mathbf{E} \times \mathbf{B})$ nonlinearity in the model, and only weak nonlinear terms associated with the density disturbance survive. These remaining weak nonlinearities are at least of the next order of smallness (in a sense of small parameter $\epsilon$ used in the derivation of the model) compared with other terms in the model equations and is not important for processes with the amplitudes considered here. These considerations allow us to model the formation of dispersive FLRs with the linearized, two-dimensional set of equations only. Additional details on the validity of the linear, two-dimensional approximation can be found in SL2.

The linear, two-dimensional, time-dependent equations of the model are:

$m_{e} n_{0} \frac{\partial v_{\| e}}{\partial t}+e n E_{\|}+\frac{\partial}{\partial z} n T_{e}=0$

$\frac{\partial n}{\partial t}+\frac{\partial}{\partial z} n_{0} v_{\| e}=0$

$\frac{m_{i} c^{2}}{B_{0}^{2}} \frac{\partial}{\partial t}\left(\frac{\partial}{\partial x} n_{0} E_{\perp}\right)+\frac{\partial j_{\|}}{\partial z}=0$.

Subsidiary relations include:

$$
\begin{array}{r}
E_{\perp}=-\frac{\partial \phi}{\partial x}, E_{\|}=-\frac{\partial \phi}{\partial z}-\frac{1}{c} \frac{\partial A}{\partial t}, \\
j_{\|} \approx-e n_{0} v_{\| e}=-\frac{c}{4 \pi} \frac{\partial^{2} A}{\partial x^{2}} .
\end{array}
$$

Here $n_{0}(x, z)$ is the background plasma density, which is chosen to provide a realistic distribution of the background Alfvén speed inside the computational domain.

To simplify the analysis, the electron temperature $T_{e}$ is taken to be constant throughout the region of interest. Thus, the background plasma model represents realistically only the distribution of the background Alfvén speed, as discussed in more detail in SL1.

In the transition region where $v_{A}=v_{T e}$ the dispersive Alfvén wave encounters electron Landau damping owing to its parallel electric field. Effects of Landau damping are not included in the reduced two-fluid model considered here. The various conditions under which Landau damping may affect dispersive Alfvén waves propagating through the transition region are discussed in SL1 and Lysak and Lotko (1996).

\subsection{Finite ionospheric conductivity model}

The geophysical processes investigated in this study are concerned with standing ULF electromagnetic waves in which the frequencies of oscillation are much smaller than $\omega_{c i}$ everywhere along the resonant field line. The parallel scale length of such solutions is much larger than the effective thickness of the ionosphere. Even for the highest harmonic considered here (mode 7), the first node in $E_{\perp}$ above the ionosphere occurs approximately at a distance of $4 R_{E}=25600 \mathrm{~km}$ along the field line (see SL2, Fig. 3) (this distance can be interpreted as a half-wave length in inhomogeneous plasma). For these waves the ionosphere can be treated as a heightintegrated substrate characterized by an anisotropic Ohm's law. Current continuity at the substrate yields the following relation between the field-aligned current density and the transverse electric field:

$j_{\|}= \pm \boldsymbol{\nabla} \cdot \boldsymbol{\Sigma} \cdot \boldsymbol{E}_{\perp}$.

Here $\boldsymbol{\Sigma}$ represents the usual height-integrated conductivity tensor (e.g., Lysak, 1990), expressed in slab geometry in terms of the Pedersen and Hall conductivities as

$\boldsymbol{\Sigma}=\left[\begin{array}{cc}\Sigma_{P} & -\Sigma_{H} \\ \Sigma_{H} & \Sigma_{P}\end{array}\right]$.

The choice of sign in Eq. (4) means that the field-aligned current is taken to be positive when $j_{\|}$flows into the ionosphere and negative in the opposite case (Lysak, 1990).

For a spatially uniform conductivity model, only the Pedersen conductivity enters Eq. (4), owing to the conservative nature of $E_{\perp}$ on the substrate. Here we will assume that the conductivity is uniform, a reasonable approximation due to the small-scale size of resonance solutions in the N-S direction [of course, the 
ionospheric conductivity may change significantly in local time (Spiro et al., 1982), but this effect is not included in the present two-dimensional model]. In this case Eq. (4) can be reduced to a second-order, ordinary differential equation for a composite function formed by the scalar potential $\phi$ and flux function $A$ :

$\frac{\partial^{2}}{\partial x^{2}}\left(\frac{c}{4 \pi} A \mp \Sigma_{P} \phi\right)=0$.

Following Lysak (1990) we introduce the Alfvén conductivity $\Sigma_{A}=c^{2} / 4 \pi v_{A}$. In the model, FLRs are excited by a boundary generator localized along the magnetic field line in the equatorial region. The numerical solutions are calculated in time until the disturbances radiated by the FLR reach the lateral boundaries. This means that the value of the scalar potential, as well as the flux function, must be equal to zero at the lateral boundaries near the ionosphere. Then, the solution of Eq. (5) with zero boundary conditions is:

$\frac{\Sigma_{A}}{\Sigma_{P}} \frac{v_{A}}{c} A \mp \phi=0$.

The reflection coefficient of electromagnetic waves is defined as $R=\left|E_{r}\right| /\left|E_{i}\right|$, where $\left|E_{r}\right|$ is the amplitude of the reflected electric field and $\left|E_{i}\right|$ is the amplitude of the incident electric field. In terms of conductivities, $R$ is given by Mallinckrodt and Carlson (1978) as

$R=\frac{\Sigma_{A}-\Sigma_{P}}{\Sigma_{A}+\Sigma_{P}}$

According to this expression, the ionosphere becomes transparent to Alfvén waves when $\Sigma_{A}=\Sigma_{P}$. In effect the magnetic field lines behave as a transmission line with characteristic propagation speed $v_{A}$ and impedance $\Sigma_{A}$; when $\Sigma_{P}=\Sigma_{A}$, the ionosphere represents a matched impedance load on the "Alfvén wave transmission line."

The Pedersen conductivity of the nightside auroral ionosphere, even at quiescent times is generally greater than 2 mho and can be as large as 25 mho during substorms (Vickrey et al., 1981; Spiro et al., 1982). The Alfvén conductivity at the topside ionosphere can be estimated from average values of the background magnetic field and density at an altitude of $0.1 R_{E}$, taking $\mathrm{O}^{+}$as the dominant ion species at this altitude. According to the truncated T87 model (Tsyganenko, 1987), $B_{0}=43958 \mathrm{nT}$ at height $0.1 R_{E}$ and latitude $67.7^{\circ}$. If $n_{0}=8000 \mathrm{~cm}^{-3}$ then $v_{A}=2679 \mathrm{~km} \mathrm{~s}^{-1}$ and $\Sigma_{A}=0.3$ mho; if $n_{0}=4000 \mathrm{~cm}^{-3}$ then $v_{A}=3788 \mathrm{~km} \mathrm{~s}^{-1}$ and $\Sigma_{A}=0.21 \mathrm{mho}$; and if $n_{0}=2000 \mathrm{~cm}^{-3}$ then $v_{A}=5358$ $\mathrm{km} \mathrm{s}^{-1}$ and $\Sigma_{A}=0.15$ mho. All these values of density are consistent with various models and observations of the auroral ionosphere (e.g., Maeda, 1975; Boehm et al., 1990). In all three cases the value of $\Sigma_{A}$ is much smaller than the value of $\Sigma_{P}$ and, therefore, typically $R \approx-1$ at the ionosphere. This means that even when the Pedersen conductivity is very small (e.g., 2 mho), Alfvén waves are mostly reflected when they reach the ionosphere. This theoretical statement is supported by the numerical simulations of the two-dimensional model performed for the first four odd harmonics FLRs.

\section{Eigenfrequencies of FLRs and numerical simulations}

In the simulations presented later, different harmonic FLRs are excited separately by the monochromatic boundary driver. By themselves monochromatic oscillations at the very-low-frequency range (about 1 $5 \mathrm{mHz}$ ) connected with field line resonances (FLRs) on the auroral magnetic field lines, are frequently measured by ground based magnetometers and HF radars (e.g., Samson et al., 1992). They may be produced by surface waves excited by plasma pressure pulses in solar wind on the dayside magnetosphere and traveling along inner plasmasheet boundary layers, or by some magnetospheric cavity modes. A monochromatic driver is used to excite only one particular harmonic in each run of the computations, because in the linear case (which is considered in the study) an interaction between different harmonics other than a simple superposition of solutions is not possible. So in order to clarify the effect of the finite ionospheric conductivity on the structure of a dispersive, nonradiative FLR, which may in general involve different harmonics, each resonance was excited separately. Realistically, we would expect that different harmonic dispersive, nonradiative FLRs could be excited simultaneously on different magnetic field lines by turbulent processes in the near-Earth magnetotail or by an impulsive perturbation on the dayside magnetopause connected with pressure pulses in solar wind. Such processes could be considered as a "broadbanded" boundary generator for the problem in hand. Unfortunately the model used in this study is incompressional, which is suitable near the resonant $\mathrm{L}$ shell, where the compressional component is weak; however, it is difficult to describe such boundary drivers realistically without compressibility.

In order to excite the FLR inside the computational domain the frequency of the driver should match one of eigenfrequencies of the system. The eigenfrequencies of dispersive, nonradiative FLRs bounded by perfectly conducting ionospheres, for the first four odd harmonics, are determined by the method described in SL2. Let us show that these eigenfrequencies could be used as rough estimations when the ionospheric conductivity is finite. We will assume that (1) the ionospheric damping rate is small compared to the resonance growth rate (otherwise, the FLR will be not observed), which, in turn, is smaller than the corresponding eigenfrequency; (2) the transverse scale size of the FLR is much smaller than the characteristic transverse scale size of the plasma inhomogeneity. In this case we may Fourier transform the linear model (1)-(3) in time and in space over $x$, and after some simple algebra reduce it to one second-order, ordinary differential equation for the Fourier transform of the flux function denoted as $\hat{A}\left(k_{x}, z, \omega\right)$ [Eq. (6) in SL2]. The ionospheric boundary condition for $\hat{A}$ is $v_{A} \partial \hat{A} / \partial z \pm\left(\Sigma_{A} / \Sigma_{P}\right) \partial \hat{A} / \partial t=0$. Because $\Sigma_{A} \ll \Sigma_{P}$ and the waves considered here are in the ULF range, the second term in the last expression can be set equal to 0 . So we get the same eigenvalue equation with the same boundary conditions as when the ionosphere is a perfect conductor (SL2). 
When the ionosphere is a perfect conductor, the eigenfrequencies of FLRs, as well as the parallel structure of the solutions, are determined by the length of the resonance magnetic field line $L_{z}$ and the background Alfvén speed profile along it. In our model we determine the resonance field line as the line passing through the midnight equatorial magnetosphere at the radial distance $9 R_{E}$. According to the truncated T87 model with $K_{p}=2^{-}, 2,2^{+}$(Tsyganenko, 1987), $L_{z}=20.7 R_{E}$ for this line. The Alfvén speed profile along this line depends on the background density and magnetic field distribution, and, in general, it is not welldefined for auroral field lines because the background density in the auroral region is highly variable, both in space and in time (Maeda, 1975; Persoon et al., 1988). In this work we will use the background Alfvén speed model introduced in SL2:

$$
\begin{aligned}
v_{A}(x, z) & =\left(1+\kappa_{1} \tanh \left(\frac{x-L_{x} / 2}{\kappa_{2}}\right)\right)^{-1 / 2} \\
& \begin{cases}v_{A 0} e^{-\left(\frac{z-z_{2}}{z_{e 1}}\right)^{2}+v_{A 1}} & \text { if } z_{1}<z<z_{2} \\
v_{A 2} e^{-\left(\frac{z-z_{2}}{z_{e 2}}\right)^{2}}+v_{A 3} & \text { if } z_{2}<z<z_{3}\end{cases}
\end{aligned}
$$

Here $L_{x}$ is the total length of the computational domain in the $x$ direction; parameters $\kappa_{1}$ and $\kappa_{2}$ control the transverse gradient in the background inhomogeneity; $z_{1}=0.1 \quad R_{E}, z_{2}=1.25 R_{E}$, and $z_{3}=10.35 R_{E}$ are coordinates of three control points on the resonance field line; $z_{e 1}=1 R_{E}$ and $z_{e 2}$ determines the location of the dispersion transition region along the resonance field line (it is adjusted for each harmonic); the constants $v_{A 0}$, $v_{A 1}, v_{A 2}$, and $v_{A 3}$ are chosen so that for each $z_{e 2}$, the function $v_{A}\left(L_{x} / 2, z\right)$ is continuous and equal to certain nominal values at three control points $\left(v_{A}\left(L_{x} / 2, z_{1}\right)\right.$ $=5000 \mathrm{~km} \mathrm{~s}^{-1}, \quad v_{A}\left(L_{x} / 2, z_{2}\right)=21462 \mathrm{~km} \mathrm{~s}^{-1}$, and $\left.v_{A}\left(L_{x} / 2, z_{3}\right)=985 \mathrm{~km} \mathrm{~s}^{-1}\right)$. Because the ambient magnetic field is assumed to be constant, this distribution in the background Alfvén speed is provided only by the background density variation:

$n_{0}(x, z)=B_{0} / 4 \pi m_{i} v_{A}^{2}(x, z)$.

For this background model the eigenfrequency of the fundamental FLR when $z_{e 2}=3.5 R_{E}$ is $6 \mathrm{mHz}$; the eigenfrequency of the third harmonic FLR when $z_{e 2}=4.75 R_{E}$ is $48 \mathrm{mHz}$; the eigenfrequency of the fifth harmonic FLR when $z_{e 2}=4.25 R_{E}$ is $72 \mathrm{mHz}$; and the eigenfrequency of the seventh harmonic FLR when $z_{e 2}=4.20 R_{E}$, is $100 \mathrm{mHz}$ (SL2, Table 2). The seventh harmonic FLR is considered here mostly for completeness and to illustrate tendencies in behavior of the higher harmonic FLRs. We do not insist that such high frequency oscillations are playing a significant role in the power spectrum of the disturbances generated in the magnetotail. At the same time, an eigenperiod of $10 \mathrm{~s}$ makes this resonance suitable as one possible explanation of flickering aurora or Pc2 pulsations, but such evaluation is beyond the scope of the study.
To understand the influence of the transverse inhomogeneity on the structure of FLR with finite ionospheric conductivity, two different transverse gradients in the background density are specified. The first is the "strong" inhomogeneity case with $\kappa_{1}=0.50$ and $\kappa_{2}=22.5 \rho_{s}$ (here $\rho_{s}=\left(T_{e} / m_{i}\right)^{1 / 2} / \omega_{c i}$ is the ion sound radius; in the model $T_{e}=100 \mathrm{eV}, B_{0}=35 \mathrm{nT}$, and hence $\omega_{c i}=3.353 \mathrm{~Hz}$ and $\rho_{s}=29 \mathrm{~km}$ ). The second, at eight times less, is the "weak" inhomogeneity case with $\kappa_{1}=0.25$ and $\kappa_{2}=90 \rho_{s} . L_{x}=150 \rho_{s}$ in the first case, and $L_{x}=450 \rho_{s}$ in the second.

The simple slab geometry of the computational domain allows a finite difference approach to be used to calculate partial derivatives in space. The computational grid for $\phi$ contains 65 levels spaced a distance $h_{z}$ along $z$, including both ionospheric boundaries. The computational grid for $A$ contains 64 levels along $z$ and starts at distance $h_{z} / 2$ above the ionospheric boundary. The difference between the last two numbers arises because $\phi$ and $A$ are calculated on shifted grids in $z$. The number of grid nodes along $x$ is the same for both $\phi$ and $A$ : 151 in the strong case and 451 in the weak case. A fourth-order predictor-corrector method with the Adams-Bashforth four-step method as a predictor and the Adams-Moulton three-step method as a corrector is used to time-advance the model equations.

The boundary condition for the electric potential on the right lateral boundary is chosen as $\phi(z)$ $=\phi_{0} \sin (\omega t) \exp \left[-\left(\left(z-L_{z} / 2\right) /\left(L_{z} / 16\right)\right)^{2}\right]$. The effective size of the boundary generator in $z$ is $L_{z} / 8=2.6 R_{E}$. Due to this particular type of symmetry, the generator excites only odd harmonics. The boundary condition for the flux function on the right lateral boundary is calculated from (1) as a surface wave solution $\left(\partial_{x}^{2}=0\right)$ : $\partial_{t} A=-c \partial_{z} \phi$. Both the electric potential and the flux function are kept equal to 0 on the left lateral boundary. On the ionospheric boundaries the flux function $A$ and scalar potential $\phi$ are connected via relation (6).

\section{Results and Discussion}

The results of simulations of the FLRs with finite conducting ionospheric boundaries, when the transverse inhomogeneity is strong, are shown in Figs. 1 and 2. Figure 1 compares the time development of maximum amplitudes of $E_{x}$ and $B_{y}$ in the computational domain for the first four odd harmonic, dispersive, nonradiative FLRs when the ionospheric conductivity is finite (broken curves) and when it is infinite (solid curves). An atypically low value of the ionospheric conductivity $\left(\Sigma_{P}=2 \mathrm{mho}\right)$ has been used to bracket the expected range of observed values. Long-dashed curves correspond to calculations when both northern and southern ionospheres are finite conducting $\left(\Sigma_{P_{N}}=\Sigma_{P_{S}}=2 \mathrm{mho}\right)$. Short-dashed curves (shown only for third harmonic and fundamental FLRs) correspond to calculations with asymmetrical ionospheric boundary conditions $\left(\Sigma_{P_{N}}\right.$ $=2$ mho and $\left.\Sigma_{P_{S}}=\infty\right)$. The asymmetry in conductivities of conjugate northern and southern auroral iono- 

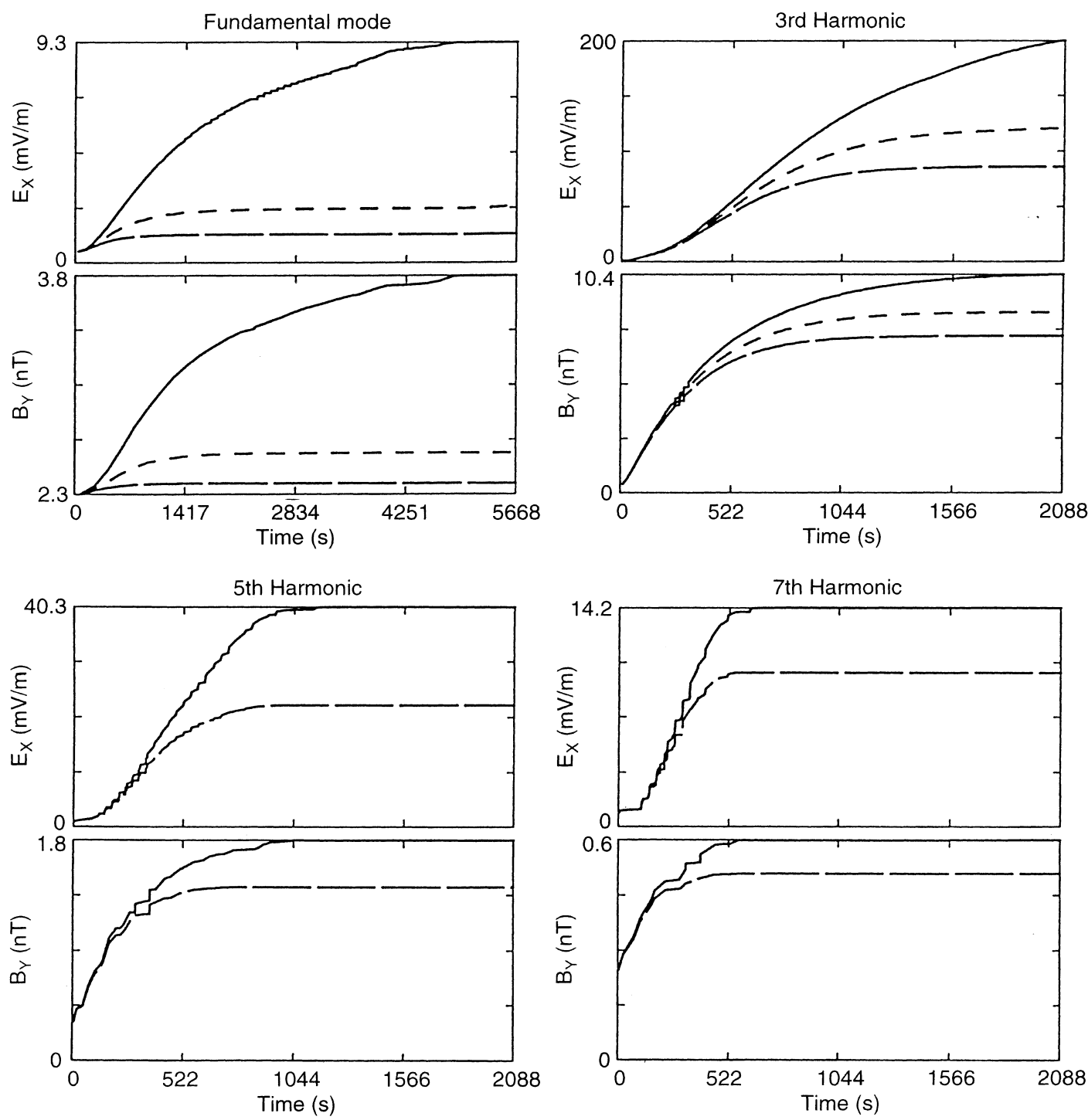

Fig. 1. Evolution of maximum amplitudes of $E_{x}$ and $B_{y}$ for the first four odd harmonic dispersive, nonradiative FLRs when the transverse plasma inhomogeneity is strong. Solid curves correspond to the case $\Sigma_{P_{S}}=\Sigma_{P_{N}}=\infty$, long-dashed curves correspond to the

case $\Sigma_{P_{S}}=\Sigma_{P_{N}}=2 \mathrm{mho}$, and short-dashed curves in panels for the fundamental and third harmonic FLRs correspond to the asymmetrical case $\Sigma_{P_{S}}=\infty$ and $\Sigma_{P_{N}}=2$ mho

spheres is typical, for example, due to the seasonal variation of the electron density.

To estimate dimensional amplitudes of calculated $E_{x}$ and $B_{y}$, we chose the amplitude of the boundary driver so that the maximum value of $E_{x}$ for the saturated, third harmonic FLR with perfectly conducting ionospheres is equal to $200 \mathrm{mV} / \mathrm{m}$ (a typical amplitude of the smallscale, large-amplitude electric field spikes measured near the ionosphere see (Mozer, 1981; Weimer and Gurnett, 1993). This same boundary driver is used for all runs to facilitate comparisons.

Figure 2 shows profiles of the $B_{y}$ for the first four odd harmonics FLRs, along the resonance field line and transverse to it, near the ionosphere. Solid lines correspond to computations with infinite ionospheric conductivity, long-dashed lines correspond to runs when $\Sigma_{P_{N}}=\Sigma_{P_{S}}=2$ mho, and short-dashed lines (in the third harmonic and fundamental panels) correspond to runs

when $\Sigma_{P_{N}}=2$ and $\Sigma_{P_{S}}=\infty$. These profiles are shown at time $5450 \mathrm{~s}$ for the fundamental mode, and at time $1318 \mathrm{~s}$ for the third, fifth, and seventh harmonics. In these moments of time, the amplitudes of the corresponding FLRs with finite ionospheric conductivities are saturated. To make the structural difference between the three curves for the fundamental FLRs more visible, the amplitude of $B_{y}$, when the conductivity of one or both ionospheres is finite, is enlarged three times.

The main qualitative conclusion to be derived from the results shown in Figs. 1 and 2 is that higherharmonic FLRs (third harmonic and up) in the strongly inhomogeneous plasma develop quickly and with appreciable amplitudes even when the ionospheric conductivity is very low. Therefore, low ionospheric conductivity cannot prevent contraction of higher-harmonic FLRs to small-scale structures where dispersive effects are important. As a result, the amplitude of the 

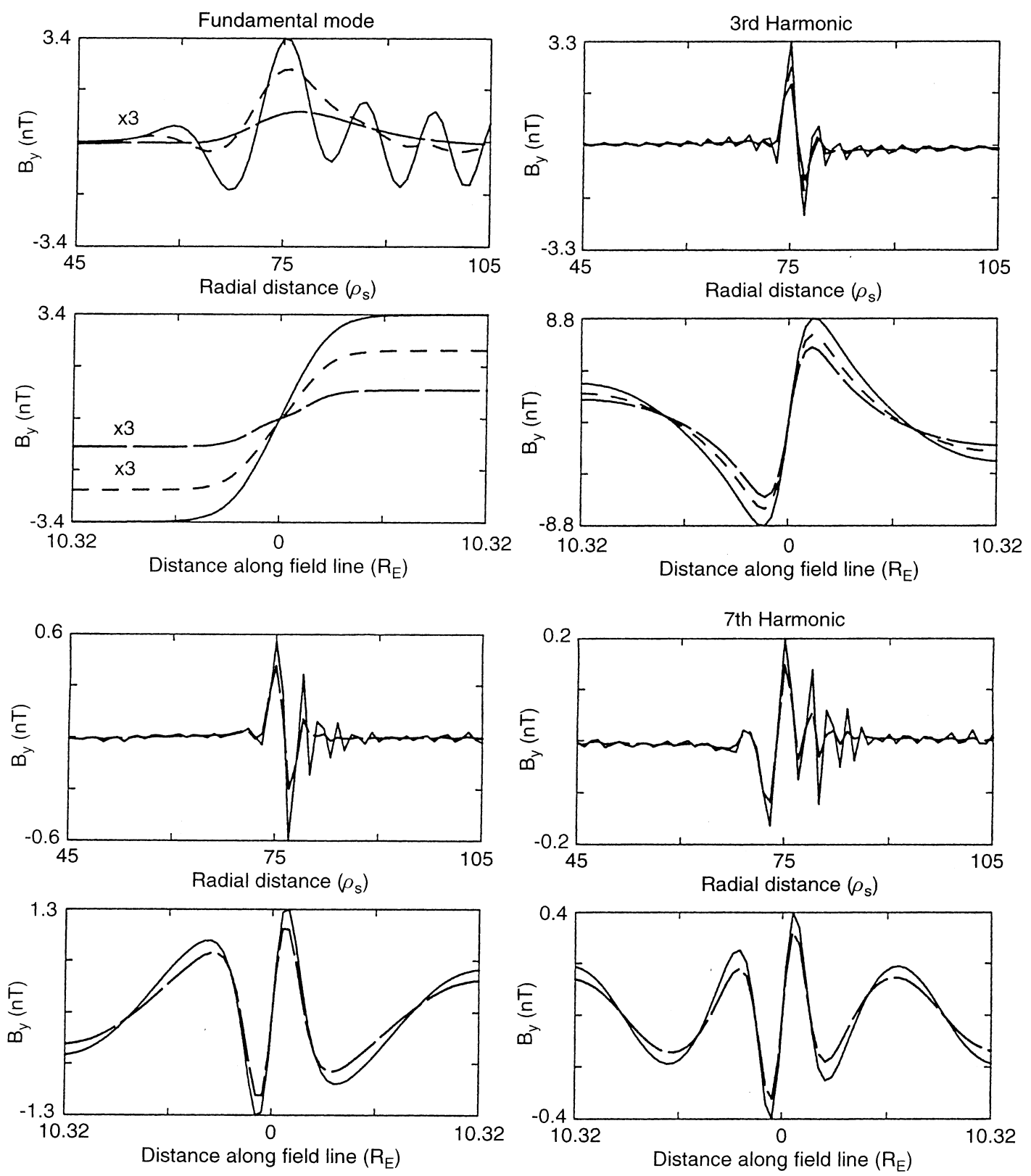

Fig. 2. Profiles of $B_{y}$ along the resonance field line and transverse to it near the ionosphere for dispersive, nonradiative FLRs at time $5450 \mathrm{~s}$ for the fundamental mode and at time $1318 \mathrm{~s}$ for the third, fifth, and seventh harmonics when the transverse plasma inhomogeneity is

strong. Solid curves correspond to the case $\Sigma_{P_{S}}=\Sigma_{P_{V}}=\infty$, longdashed curves correspond to the case $\Sigma_{P_{S}}=\Sigma_{P_{N}}=2 \mathrm{mho}$, and shortdashed curves in panels for the fundamental and third harmonic FLRs correspond to the asymmetrical case $\Sigma_{P_{S}}=\infty$ and $\Sigma_{P_{N}}=2 \mathrm{mho}$

electromagnetic field in these resonances can be easily measured by low-altitude satellites as small-scale, largeamplitude spikes. As discussed in SL2, the parallel electric field in these harmonic resonances is sufficient to accelerate electrons to several kilovolts in energy.

The fundamental mode is more strongly affected by the low ionospheric conductivity. Even when the conductivity of only one of the two ionospheres is low (e.g. $\Sigma_{P_{N}}=2$ mho and $\Sigma_{P_{S}}=\infty$ ) the fundamental FLR saturates at the "predispersive" level. That is, the resonance stabilizes as a fairly broad (compared with higher-harmonic cases), low-amplitude electromagnetic disturbance because the finite ionospheric conductivity damps the fundamental mode more strongly than the harmonics. This result agrees qualitatively with calculations of the ionospheric damping rate for different harmonics given by Newton et al. (1978). Apart from its larger ionospheric damping rate, there are at least two additional reasons why the fundamental FLR saturates at a low level. First, the growth rate of the fundamental mode, even when the ionosphere is a perfect conductor, is slower than that of the higher harmonic modes (solid curves in Fig. 1). As a consequence, the fundamental mode loses energy through the conducting ionosphere almost as fast as it absorbs it from the boundary generator. The second reason is that the transverse scale 

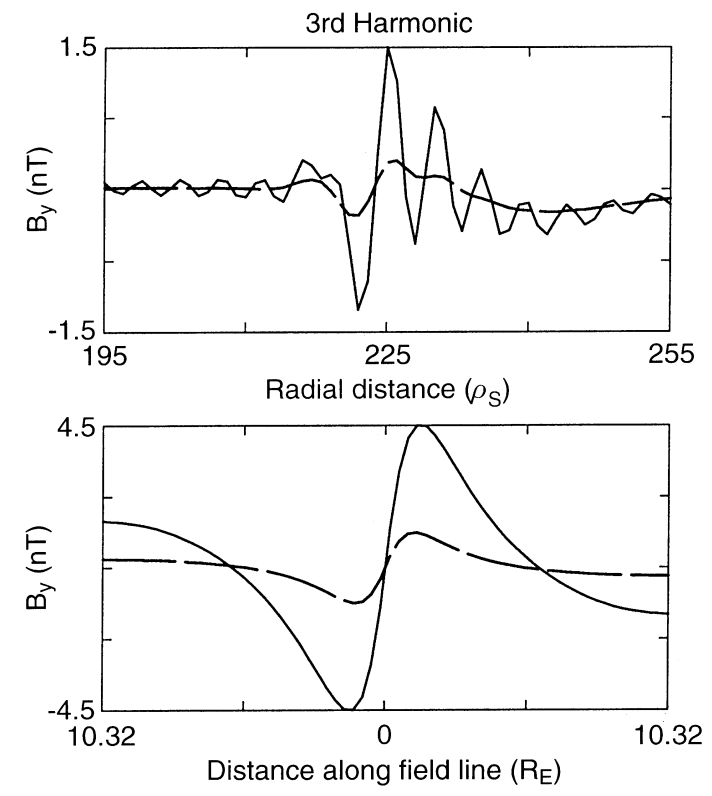

Fig. 3. Profiles of $B_{y}$ along the resonance field line (bottom) and transverse to it near the ionosphere (top) for the third harmonic dispersive, nonradiative FLR at time $5019 \mathrm{~s}$ when the transverse plasma inhomogeneity is weak. Solid curves correspond to the case $\Sigma_{P_{S}}=\Sigma_{P_{N}}=\infty$, long-dashed curves correspond to the case $\Sigma_{P_{S}}=\Sigma_{P_{N}}=2$ mho

size of the fundamental FLR layer is larger than that of the higher harmonics (Fig. 2). Therefore the fundamental FLR interacts with the ionosphere over a larger spatial region compared with higher harmonic FLRs.

Our calculations show that asymmetrical ionospheric boundary conditions $\left(\Sigma_{P_{N}}=2\right.$ mho and $\left.\Sigma_{P_{S}}=\infty\right)$ do not produce any significant asymmetry in the distribution of the electromagnetic field along the resonance field line (Fig. 2). We have considered this question only for the fundamental and third harmonic FLRs because these two are the most sensitive to ionospheric boundary conditions. As a whole, in both cases, the solutions are closer to the situation when both ionospheres are finite conducting $\left(\Sigma_{P_{N}}=\Sigma_{P_{S}}=2 \mathrm{mho}\right)$.

Relation (7) shows that the Alfvén wave reflection coefficient at the ionosphere becomes smaller as $\Sigma_{A}$ is increased. (Recall that typically $\Sigma_{A} \ll \Sigma_{P}$ near the topside ionosphere.) To quantify this statement two runs were made for the third harmonic FLR for fixed ionospheric conductivity $\left(\Sigma_{P}=2 \mathrm{mho}\right)$, but for two different values of the Alfvén conductivity. In one case, $\Sigma_{A}=0.16$ mho $\left(v_{A}=5000 \mathrm{~km} \mathrm{~s}^{-1}\right)$ at the ionospheric boundary; in the second case, $\Sigma_{A}=0.40$ mho $\left(v_{A}=2000\right.$ $\mathrm{km} / \mathrm{s}$ ). For the higher Alfvén conductivity case, the saturation amplitude of $E_{x}$ decreases to $46 \%$ of its value in the lower $\Sigma_{A}$ case; the corresponding saturation amplitude of $B_{y}$ decreases to $18 \%$. Higher $\Sigma_{A}$ brings the ionosphere closer to a matched impedance load (in the "transmission line" analogy), so more energy is lost to the ionosphere even though its conductivity is fixed. This behavior indicates that the saturation amplitude of the FLR is sensitive to the effective altitude of the ionospheric boundary. For the case when $\Sigma_{P}=\infty$, the FLR amplitude is essentially independent of the Alfvén speed near the ionosphere.

The importance of a strong, localized transverse inhomogeneity in the background Alfvén speed for development of small-scale, large-amplitude FLRs was discussed in SL1 on the basis of results obtained when the ionosphere is treated as a perfect conductor. To illustrate how the transverse gradient of the background Alfvén speed can affect the FLR when the ionospheric conductivity is finite, two additional sets of computations were performed for the third harmonic, nonradiative FLR when the background inhomogeneity is weak. In one case $\Sigma_{P}$ was set equal to $2 \mathrm{mho}$, in another the ionospheres were assumed to be perfect conductors. Figure 3 shows the corresponding profiles of $B_{y}$ along the resonant field line (bottom panel) and in the transverse direction near the ionosphere (top panel) at time $5019 \mathrm{~s}$. Because the time scale for the FLR to develop dispersive structure increases as the scale size of the transverse inhomogeneity in the background Alfvén speed increases (Inhester, 1987), the FLR takes longer to develop appreciable amplitude for the weakly inhomogeneous case (cf. $5019 \mathrm{~s}$ with $1318 \mathrm{~s}$ for the strongly inhomogeneous case considered in Figs. 1 and 2).

The main conclusion to be derived from Fig. 3 is that a low ionospheric conductivity can prevent even higher harmonic FLRs from developing small-scale, dispersive, large-amplitude, electromagnetic structure when the transverse variation in the background density is relatively gradual. This result emphasizes the special rope of strong transverse density gradient in the development of dispersive FLRs. The existence of strong transverse density gradients in the lower magnetosphere at the edges of "auroral plasma cavities" (Persoon et al., 1988) may be especially important in this regard.

According to the results shown in Fig. 1, the saturation time of the higher harmonic FLRs is relatively short and depends only weakly on $\Sigma_{P}$. Consequently, when the background inhomogeneity is strong, the higher harmonic FLRs develop quickly even when the ionospheric conductivity is low. As time proceeds, enhanced ionization caused by accompanying precipitating electrons is expected to increase the ionospheric conductivity, providing favorable conditions for lower harmonic FLRs, including the fundamental. This nonlinear effect (which is not included in the model) suggests that the time-evolution of an initially undisturbed, auroral ionosphere, under the influence of a magnetospheric generator, will first form small-scale, intense, higher-frequency oscillations corresponding to the higher harmonic FLRs. The formation of systems of bright and narrow auroral arcs during the early phase of substorms (Xu et al., 1993) may be consistent with this scenario. For this situation, the height-integrated conductivity would be better modeled by an empirical relation (e.g. Spiro et al., 1982) that allows the conductivity to increase with increasing intensity of upward field-aligned current density.

At the same time, the higher-harmonic FLRs, that may lead to the formation of small-scale, intense discrete auroral arcs, are not detectable by the ground magne- 
tometers, a consequence of the fact that small-scale magnetic fields are shielded from the ground by the conducting ionosphere: "... the signal at a ground station is given by the integrated effect of Hall currents flowing in a region of the ionosphere comparable in scale to the height of the E-region above the ground $(\sim 120 \mathrm{~km}) "$ (Southwood and Hughes, 1983). This observational constraint may be one of the main reasons why mostly fundamental FLRs, and rarely harmonics, are registered on the ground.

\section{Summary}

Summarizing results presented in the paper, the following main conclusions can be drawn:

1. The low ionospheric conductivity does not change the fine structure of the dispersive, nonradiative, higherharmonic FLRs when the transverse background inhomogeneity of the plasma is strong enough. The fundamental FLR is the most strongly affected by the low ionospheric conductivity. When the conductivity is too low, the fundamental resonance develops only very broad, nondispersive electromagnetic structure.

2 . The gradient in the transverse background inhomogeneity is the key parameter in the development of FLR layers. When the transverse inhomogeneity is weak, and the ionospheric conductivity is low, even the third harmonic FLR can saturate at the predispersive level.

3. Even significant differences in northern and southern ionospheric conductivities does not produce asymmetry in the resonance solutions. This finding means that the parallel inhomogeneity of the plasma is more important than the ionospheric boundary conditions in determining the parallel structure of dispersive, nonradiative FLRs.

Acknowledgements. The research was supported in part by NASA under grants NAG5-2252, NAG5-1098, and NAGW-1824, and by the Thayer School of Engineering, Dartmouth College, Hanover, NH, USA

Topical Editor K.-H. Glaßmeier thanks J.-C. Samson and V. Pilipenko for their help in evaluating this paper.

\section{References}

Akasofu, S.-I., Past, present and future of substorm research, Proc. Second International Conference on Substorms, 13, 1994.

Bennett, E. L., M. Temerin, and F. S. Mozer, The distribution of auroral electrostatic shocks below 8000-km altitude, J. Geophys. Res., 88, 7107, 1983.

Boehm, M. H., C. W. Carlson, J. P. McFadden, J. H. Clemmons, and F. S. Mozer, High-resolution sounding rocket observations of large-amplitude Alfvén waves, J. Geophys. Res., 95, 12, 157, 1990

Chen, L., and A. Hasegawa, A theory of long-period magnetic pulsations, 1 . Steady state excitation of field line resonance, $J$. Geophys. Res., 79, 1024, 1974.

Chmyrev, V. M., S. V. Bilichenko, O. A. Pokhotelov, V. A. Marchenko, V. I. Lazarev, A. V. Streltsov, and L. Stenflo, Alfvén vortices and related phenomena in the ionosphere and the magnetosphere, Phys. Scr., 38, 841, 1988.

Goertz, C. K., and R. W. Boswell, Magnetosphere-ionosphere coupling, J. Geophys. Res., 84, 7239, 1979.

Greenwald, R. A., and A. D. M. Walker, Energetics of long period resonant hydromagnetic waves, Geophys. Res. Lett., 7, 745, 1980.

Hasegawa, A., Particle acceleration by MHD surface wave and formation of aurora, J. Geophys. Res., 81, 5083, 1976.

Hughes, W. J., and R. J. L. Grard, A second harmonic geomagnetic field line resonance at the inner edge of the plasma sheet: GOES 1, ISEE 1, and ISEE 2 observations, J. Geophys. Res., 89, 2755, 1984.

Inhester, B., Numerical modeling of hydromagnetic wave coupling in the magnetosphere, J. Geophys. Res., 92, 4751, 1987.

Kadomtsev, B. B., Plasma turbulence, Academic Press, New York, 1965.

Lysak, R. L., Electromagnetic coupling of the magnetosphere and ionosphere, Space Sci. Rev., 52, 33, 1990.

Lysak, R. L., and C. Carlson, Effect of microscopic turbulence on magnetosphere-ionosphere coupling, Geophys. Res. Lett., 8, 269, 1981.

Lysak, R. L., and W. Lotko, On the kinetic dispersion relation for shear Alfvén waves, J. Geophys. Res., 101, 5085, 1996.

Maeda, K., A calculation of auroral hiss with improved models for geoplasma and magnetic field, Planet. Space Sci., 23, 843, 1975.

Mallinckrodt, A. J., and C. W. Carlson, Relations between transverse electric fields and field-aligned currents, J. Geophys. Res., 83, 1426, 1978.

Mozer, F. S., ISEE-1 observations of electrostatic shocks on auroral zone field lines between 2.5 and 7 earth radii, Geophys. Res. Lett., 8, 823, 1981.

Newton, R. S., D. J. Southwood and W. J. Hughes, Damping of geomagnetic pulsations by the ionosphere, Planet. Space Sci., 26, 201, 1978.

Persoon, A. M., D. A. Gurnett, W. K. Peterson, J. H. Waite, Jr., J. L. Burch, and J. L. Green, Electron density depletions in the nightside auroral zone, J. Geophys. Res., 93, 1871, 1988.

Samson, J. C., D. D. Wallis, T. J. Hughes, F. Greutzberg, J. M. Ruohoniemi, and R. A. Greenwald, Substorm intensifications and field line resonances in the nightside magnetosphere, $J$. Geophys. Res., 97, 8495, 1992.

Southwood, D. J., Some features of field line resonances in the magnetosphere, Planet. Space Sci., 22, 483, 1974.

Southwood, D. J., and W. J. Hughes, Theory of hydromagnetic waves in the magnetosphere, Space Sci. Rev., 35, 301, 1983.

Spiro, R. W., P. H. Reiff, and L. J. Maher, Precipitating electron energy flux and auroral zone conductances: an empirical model, J. Geophys. Res., 87, 8215, 1982.

Streltsov, A., and W. Lotko, Dispersive field line resonances on auroral field lines, J. Geophys. Res., 100, 19, 457, 1995.

Streltsov, A., and W. Lotko, The fine structure of dispersive, nonradiative field line resonance layers, J. Geophys. Res., 101, 5343, 1996.

Tsyganenko, N. A., Global quantitative models of the geomagnetic field in the cislunar magnetosphere for different disturbance levels, Planet. Space Sci., 35, 1347, 1987.

Vickrey, J. F., R. R. Vondrak, and S. J. Matthews, The diurnal and latitudinal variations of auroral-zone ionospheric conductivity, J. Geophys. Res., 86, 65, 1981.

Wei, C. Q., J. C. Samson, R. Rankin, and P. Frycz, Electron inertial effects on geomagnetic field line resonances, J. Geophys. Res., 99, $11,265,1994$.

Weimer, D. R. and D. A. Gurnett, Large-amplitude auroral electric fields measured with DE-1, J. Geophys. Res., 98, 13,557, 1993.

Xu, B.-L., J. C. Samson, W. W. Liu, F. Creutzberg, and T. J. Hughes, Observations of optical aurora modulated by resonant Alfvén waves, J. Geophys. Res., 98, 11, 531, 1993. 